\title{
A GENERALIZED PALEY-WIENER-ZYGMUND INTEGRAL AND ITS APPLICATIONS
}

\author{
CHULL PARK ${ }^{1}$
}

1. Introduction. Let $Y_{N}$ denote the product space $\prod_{j=1}^{N}\left[a_{j}, b_{j}\right]$ for $N=1,2, \cdots$ and $p=\left(p_{1}, \cdots, p_{N}\right)$ any point in $Y_{N}$. The $(N-$ dimensional) Yeh-Wiener space $C\left(Y_{N}\right)=C_{N}$ is the set of all realvalued continuous functions $f(p)$ on $Y_{N}$ such that $f(p)=0$ if $p_{j}=a_{j}$ for some $1 \leqq j \leqq N$. Thus $C_{N}$ becomes the Wiener space $C_{W}$ if $Y_{N}$ $=[0,1]$. (For the measure of this space consult with [3] and [5].) The definition of "bounded variation" and that of the ordinary R-S (Riemann-Stieltjes) integral in $Y_{N}$ are found in [5].

It is known that if $h \in L^{2}\left(Y_{N}\right)$ and $h_{n}$ the $n$th partial sum of the Fourier-series of $h$ with respect to a C.O.N. set $\left\{\alpha_{k}\right\}$ with each $\alpha_{k}$ of B.V. on $Y_{N}$, then the P.W.Z. (Paley, Wiener, Zygmund) integral

$$
\int_{Y_{N}} h d^{*} f \equiv \lim _{n} \int_{Y_{N}} h_{n} d f, \quad \text { where } \int_{Y_{Y}} h_{n} d f
$$

is the ordinary R-S integral, exists for almost all $f$ in $C_{N}$, and that it is essentially independent of the particular choice of the C.O.N. set on $Y_{N}$, and also that the integral is essentially consistent with the ordinary R-S integral if $h$ is of B.V. on $Y_{N}$. The case when $N=1$ was discussed in [1], for $N=2$ in [2], and for more general cases in [3].

Some basic questions that arise here are these:

If $h \in L^{2}\left(Y_{N}\right)$, does there exist

$$
\int_{Y_{N}} h f d^{*} f
$$

for almost all $f$ in $C_{N}$ ? If it does, is it essentially consistent for various C.O.N. sets? The answer to these questions is much deeper than it

Presented to the Society, November 11, 1968 under the title An extension of PaleyWiener-Zygmund integral and on January 26, 1969 under the title Applications of Paley-Wiener-Zygmund integral; received by the editors May 20, 1968 and, in revised form, March 26, 1969.

1 This paper is a generalization of a portion of the author's doctoral dissertation under Professor R. H. Cameron at the University of Minnesota. The research was supported in part by the Air Force Office of Scientific Research, Office of Aerospace Research, United States Air Force, Grant No. AFOSR-381-65 and No. AFOSR381-66. Further research on it was done at Miami University. 
appears to be. Thus, if the above quoted properties for the P.W.Z. integral hold for all $f$ instead of almost all, the answer would be affirmative, since $h \in L^{2}\left(Y_{N}\right)$ and $f \in C_{N}$ imply $h f \in L^{2}\left(Y_{N}\right)$. However, since the P.W.Z. integral does not exist for all $f$ in $C_{N}$, it implies nothing about (1.1). It is easy to see that $\int_{Y_{N}} h g d^{*} f$ must exist for almost all $(f, g)$ in $C_{N} \times C_{N}$. But the set where $f=g$ is a null set in $C_{N} \times C_{N}$, and thus the almost everywhere existence of $\int_{Y_{N}} h g d^{*} f$ does not imply that (1.1) exists anywhere.

Using other techniques, however, we shall show that for a large class of C.O.N. sets, the existence of (1.1) for almost all $f$ in $C_{N}$ can be proved, and the equality of the values of (1.1) for any two C.O.N. sets of the class holds for almost all $f$ in $C_{N}$. Existence theorem but no consistency theorem was obtained by Colson [4] for the corresponding problem for functions of one variable under somewhat similar condition on C.O.N. sets.

\section{Statement of main results.}

Theorem 1. Let $h \in L^{2}\left(Y_{N}\right)$ and let $\left\{\alpha_{k}\right\}$ be a real-valued C.O.N. set of functions on $Y_{N}$ each of which is of B.V. on $Y_{N}$. Furthermore let

$$
\lim _{n} \sum_{k=1}^{n} \alpha_{k}(p) \int_{A(p)} \alpha_{k}(q) d \mu_{N}(q)=2^{-N}
$$

in $L^{2}$-sense on $Y_{. N}$, where $A(p)=\prod_{j=1}^{N}\left[a_{j}, p_{j}\right]$ for each $p=\left(p_{1}, \cdots, p_{N}\right)$ in $Y_{N}$ and $\mu_{N}$ the Lebesgue measure in $R_{N}$. For each $f$ in $C_{N}$ let $(h f)_{n}$ denote the nth partial sum of the Fourier series of hf with respect to $\left\{\alpha_{k}\right\}$. Then l.i.m. $\int_{Y_{N}}(h f)_{n} d f$ exists in $L^{2}$-sense on $C_{N}$.

Theorem 2. If $h,\left\{\alpha_{k}\right\}$, and $(h f)_{n}$ are as in Theorem 1 , then

$$
\int_{Y_{N}} h f d^{*} f \equiv \lim _{n} \int_{Y_{N}}(h f)_{n} d f
$$

exists for almost all $f$ in $C_{N}$.

Theorem 3. If $h \in L^{2}\left(Y_{N}\right)$, then $\int_{Y_{N}} h f d^{*} f$ is essentially consistent with different choices of C.O.N. sets in the sense that if $\left\{\alpha_{k}\right\}$ and $\left\{\beta_{k}\right\}$ are two different C.O.N. sets satisfying the conditions of Theorem 1 , then

$$
\{\alpha\} \int_{Y_{N}} h f d^{*} f=\{\beta\} \int_{Y_{N}} h f d^{*} f
$$

for almost all $f$ in $C_{N}$. 
Corollary. If $h \in L^{2}\left(Y_{N}\right)$, then

$$
\begin{gathered}
E_{N}^{f}\left[\int_{Y_{N}} h f d^{*} f\right]^{2}=\frac{1}{4} \int_{Y_{N}} h^{2}(p) \prod_{j=1}^{N}\left(p_{j}-a_{j}\right) d \mu_{N}(p) \\
+4^{-(N+1)}\left(\int_{Y_{N}} h d \mu_{N}\right)^{2},
\end{gathered}
$$

where $E_{N}^{f}[F(f)]$ denotes the integral $\int_{C_{N}} F(f) d m_{N}(f), m_{N}$ being the YehWiener measure in $C_{N}$, and $p=\left(p_{1}, \cdots, p_{N}\right), \prod_{j=1}^{N}\left(p_{j}-a_{j}\right)$ $\equiv\left(p_{1}-a_{1}\right) \cdots\left(p_{N}-a_{N}\right)$.

THeorem 4. If $h \in L^{2}\left(Y_{N}\right), f$ is absolutely continuous on $Y_{N}$, and if $\partial^{N} f / \partial p_{1} \cdots \partial p_{N} \in L^{2}\left(Y_{N}\right)$, then the P.W.Z. integral $\int_{Y_{N}} h d^{*} f$ converges and is equal to the Lebesgue integral $\int_{Y_{N}} h \cdot\left(\partial^{N} f / \partial p_{1} \cdots \partial p_{N}\right) d \mu_{N}$.

COROLLARY. If h and $f$ are as in the theorem, then

$$
\int_{Y_{N}} h f^{n} d^{*} f=\int_{Y_{N}} h f^{n}\left(\partial^{N} f / \partial p_{1}, \cdots, \partial p_{N}\right) d \mu_{N} \text { for } n=0,1,2, \cdots
$$

THEOREM 5. Let $h(t)$ be absolutely continuous and $h^{\prime}(t)$ of class $L^{2}[0,1]$. Then $\int_{0}^{1} h f d^{*} f=1 / 2 \int_{0}^{1} h(t) d\left[f^{2}(t)\right]$ for almost all $f \in C_{W}$.

TheOREM 6. Let $\left\{\alpha_{k}(t)\right\}$ and $\left\{\beta_{k}(t)\right\}$ be the C.O.N. cosine and sine functions on $[0,1]$ respectively, i.e., $\alpha_{0}(t)=1, \alpha_{k}(t)=2^{1 / 2} \cos k \pi t$, $k=1,2, \cdots$, and $\beta_{k}(t)=2^{1 / 2} \sin k \pi t, k=1,2, \cdots$. For each $f \in C_{W}$ let $a_{k}=a_{k}(f)=\int_{0}^{1} f(t) \alpha_{k}(t) d t$ and $b_{k}=b_{k}(f)=\int_{0}^{1} f(t) \beta_{k}(t) d t$. Then for almost all $f$ in $C_{W}$ we have

(i) $\sum_{k=1}^{\infty} k a_{k} b_{k}=-f^{2}(1) /(2 \pi)$,

(ii) $\sum_{k=1}^{\infty}(-1)^{k} a_{k}=2^{-1 / 2}\left[f(1)-\int_{0}^{1} f(t) d t\right]$,

(iii) $\sum_{k=1}^{\infty} a_{2 k}=\left(2^{1 / 2} / 4\right)\left[f(1)-2 \int_{0}^{1} f(t) d t\right]$,

(iv) $\sum_{k=1}^{\infty} a_{k}=\left(-2^{1 / 2} / 2\right) \int_{0}^{1} f(t) d t$.

Corollary. The Fourier cosine series converges to zero at the origin for almost all $f$ in $C_{W}$.

Remarks. 1. The convergence of (i), (ii), (iii), and (iv) in the theorem holds true for every absolutely continuous functions $f$ on $[0,1]$ with $f^{\prime} \in L^{2}[0,1]$ by virtue of the corollary to Theorem 4 with $N=1$. 
2. The corollary is a good contrast to DuBois-Reymond's Theorem (see [9]) which asserts the existence of a continuous function whose Fourier series does not converge at the origin.

3. Complete orthonormal sets satisfying our conditions. (I) Let $\left\{\alpha_{k}(x)\right\}$ be the C.O.N. cosine functions on $[a, b]$, i.e.,

$$
\begin{array}{r}
\alpha_{1}(x)=\frac{1}{\sqrt{b-a}}, \quad \alpha_{k}(x)=\sqrt{\frac{2}{b-a}} \cos \frac{(k-1) \pi(x-a)}{b-a}, \\
k=2,3, \ldots .
\end{array}
$$

Then

$$
\lim _{n} \sum_{k=1}^{n} \alpha_{k}(x) \int_{a}^{x} \alpha_{k}(s) d s=\frac{1}{2}
$$

in $L^{2}$-sense on $[a, b]$.

A straightforward computation leads to

$$
\lim _{n} \int_{a}^{b}\left[\sum_{k=1}^{n} \alpha_{k}(x) \int_{a}^{x} \alpha_{k}(s) d s-\frac{1}{2}\right]^{2} d x=0 .
$$

Direct computations also show that the C.O.N. sine functions, the C.O.N. trigonometric functions, and the C.O.N. set obtained from Legendre polynomials satisfy (3.1). More generally we give the following, whose proof is given in [2].

(II) Let $\left\{u_{k}(x)\right\}$ be the C.O.N. characteristic functions of the Sturm-Liouville system

$$
\begin{aligned}
& \left.\left[p(x) u^{\prime}(x)\right]^{\prime}+q(x) \rho-r(x)\right] u(x)=0 \\
& u^{\prime}(a)-\lambda \cdot u(a)=0, \quad u^{\prime}(b)+\mu \cdot u(b)=0
\end{aligned}
$$

where $p(x), q(x)$, and $r(x)$ are continuous and positive on $[a, b]$, and let each $u_{k}(x)$ correspond to the characteristic number $\rho_{k}$ with $\rho_{k} \uparrow$. Then it follows that

$$
\lim _{n} \sum_{k=1}^{n} u_{k}(x) \int_{a}^{x} u_{k}(t) d t=\frac{1}{2} \quad \text { on }[a, b] .
$$

(III) The C.O.N. sets obtained above are directly connected with the satisfactory C.O.N. sets for the definition of $\int_{Y_{N}} h f d^{*} f$ as follows:

For each $j, 1 \leqq j \leqq N$, let $\left\{\alpha_{k}^{(j)}\right\}$ be a C.O.N. set of functions satisfying (3.3) on $\left[a_{j}, b_{j}\right]$. Then 


$$
\begin{aligned}
\lim _{n k_{1}, \ldots, k_{N}=1} \sum_{k_{1}}^{n} \alpha_{k_{1}}^{(1)}\left(p_{1}\right) & \cdots \alpha_{k_{N}}^{(N)}\left(p_{N}\right) \\
& \cdot \int_{A(p)} \alpha_{k_{1}}^{(1)}\left(q_{1}\right) \cdots \alpha_{k_{N}}^{(N)}\left(q_{N}\right) d \mu_{N}(q)=2^{-N} .
\end{aligned}
$$

This follows immediately by observing that

$$
\begin{array}{r}
\sum_{k_{1}, \cdots, k_{N}=1}^{n} \alpha_{k_{1}}^{(1)}\left(p_{1}\right) \cdots \alpha_{k_{N}}^{(N)}\left(p_{N}\right) \int_{A(p)} \alpha_{k_{1}}^{(1)}\left(q_{1}\right) \cdots \alpha_{k}^{(N)}\left(q_{N}\right) d \mu_{N}(q) \\
=\prod_{j=1}^{N}\left[\sum_{k_{j}=1}^{n} \alpha_{k_{j}}^{(j)}\left(p_{j}\right) \int_{a_{j}}^{p_{j}} \alpha_{k_{j}}^{(j)}\left(q_{j}\right) d q_{j}\right] .
\end{array}
$$

4. Necessary lemmas. For the proof of the theorems we need the following lemmas.

Lemma 1 (P.W.Z. Theorem). Let $\alpha_{j}, j=1,2, \cdots, n$, be an orthonormal set each of which is of B.V. on $Y_{N}$. Let $F\left(u_{1}, \cdots, u_{n}\right)$ be a Lebesgue-measurable function on $R_{n}$. Then

$$
\begin{aligned}
E_{N}^{f}\left[F\left(\int_{Y_{N}} \alpha_{1} d f, \cdots, \int_{Y_{N}} \alpha_{n} d f\right)\right] & \\
& =\pi^{-n / 2} \int_{R_{n}} F\left(u_{1}, \cdots, u_{n}\right) \exp \left(-\sum_{j=1}^{n} u_{j}^{2}\right) d u_{1} \cdots d u_{n}
\end{aligned}
$$

in the sense the existence of one side implies the existence of the other and the equality. (See [6].)

Lemma 2. If $h_{j}, j=1,2,3,4$, are functions of B.V. on $Y_{N}$, then

$$
\begin{aligned}
E_{N}^{f}\left[\prod_{j=1}^{4} \int_{Y_{N}} h_{j} d f\right]=\frac{1}{4}\left[\int_{Y_{N}} h_{1} h_{2} d \mu_{N} \cdot \int_{Y_{N}} h_{3} h_{4} d \mu_{N}\right. \\
+\int_{Y_{N}} h_{1} h_{3} d \mu_{N} \cdot \int_{Y_{N}} h_{2} h_{4} d \mu_{N} \\
\left.+\int_{Y_{N}} h_{1} h_{4} d \mu_{N} \cdot \int_{Y_{N}} h_{2} h_{3} d \mu_{N}\right]
\end{aligned}
$$

This lemma can be established by othonormalizing the $h_{j}$ and then using Lemma 1.

Lemma 3 (Bearman's Theorem). Let $F(f, g)$ be an integrable functional on $C_{N} \times C_{. N}$. Then 


$$
E_{N}^{o}\left(E_{N}^{f}[F(f, g)]\right)=E_{N}^{o}\left(E_{N}^{f}[F(f \cos \theta-g \sin \theta, f \sin \theta+g \cos \theta)]\right) .
$$

Bearman [7] has proved this theorem for $N=1$. The validity of this theorem for arbitrary $N$ is immediate.

5. Proof of Theorem 1. By the definition of $(h f)_{n}$ we have

$$
\int_{Y_{N}}(h f)_{n} d f=\sum_{k=1}^{n}\left(\int_{Y_{N}} h f \alpha_{k} d \mu_{N}\right) \int_{Y_{N}} \alpha_{k} d f,
$$

and for $n>m$

$$
\begin{aligned}
I_{m, n}= & E_{N}^{f}\left[\int_{Y_{N}}(h f)_{n} d f-\int_{Y_{N}}(h f)_{m} d f\right]^{2} \\
= & \sum_{j, k=m}^{n} E_{N}^{f}\left[\int_{Y_{N}} h f \alpha_{j} d \mu_{N} \cdot \int_{Y_{N}} h f \alpha_{k} d \mu_{N} \cdot \int_{Y_{N}} \alpha_{j} d f \cdot \int_{Y_{N}} \alpha_{k} d f\right] \\
= & \sum_{j, k=m}^{n} E_{N}^{f}\left[\int_{Y_{N}^{2}} h(p) f(p) \alpha_{j}(p) h(q) f(q) \alpha_{k}(q)\left(\int_{Y_{N}} \alpha_{j} d f\right)\right. \\
& \left.\cdot\left(\int_{Y_{N}} \alpha_{k} d f\right) d \mu_{2 N}\right] .
\end{aligned}
$$

By Fubini's theorem

$$
\begin{aligned}
I_{m, n}=\sum_{j, k=m}^{n} \int_{Y_{N}^{2}} h(p) \alpha_{j}(p) h(q) \alpha_{k}(q) \\
\cdot\left\{E_{N}^{j}\left[f(p) f(q)\left(\int_{Y_{N}} \alpha_{j} d f\right)\left(\int_{Y_{N}} \alpha_{k} d f\right)\right]\right\} d \mu_{2 N} .
\end{aligned}
$$

Since $f \in C_{N}$ and $p, q \in Y_{N}$, we may write $f(p)=\int_{a_{N}}^{p_{N}} \cdots \int_{a_{1}}^{p_{1}} d f=\int_{A(p)} d f$ and $f(q)=\int_{A(q)} d f$, where $A(p)=\prod_{j=1}^{N}\left[a_{j}, p_{j}\right]$. We define a function $\xi(p, s)$ on $Y_{N}^{2}$ by

$$
\begin{array}{rlrl}
\xi(p, s) & =1 \quad \text { if } s_{i} \leqq p_{i} \quad \text { for } i=1, \cdots, N \\
& =0 & \text { otherwise. } &
\end{array}
$$


Then

$$
f(p)=\int_{Y_{N}} \xi(p, s) d f(s), \quad f(q)=\int_{Y_{N}} \xi(q, s) d f(s) .
$$

Using these expressions and then applying Lemma 3, we obtain

$$
\begin{aligned}
E_{N}^{f}\left[f(p) f(q)\left(\int_{Y_{N}} \alpha_{j} d f\right)\left(\int_{Y_{N}} \alpha_{k} d f\right)\right] \\
=\frac{1}{4}\left[\int_{Y_{N}} \xi(p, s) \xi(q, s) d \mu_{N}(s) \cdot \int_{Y_{N}} \alpha_{j} \alpha_{k} d \mu_{N}\right. \\
\quad+\int_{Y_{N}} \xi(p, s) \alpha_{j}(s) d \mu_{N}(s) \cdot \int_{Y_{N}} \xi(q, s) \alpha_{k}(s) d \mu_{N}(s) \\
\left.\quad+\int_{Y_{N}} \xi(p, s) \alpha_{k}(s) d \mu_{N}(s) \cdot \int_{Y_{N}} \xi(q, s) \alpha_{j}(s) d \mu_{N}(s)\right] \\
=\frac{1}{4}\left\{\delta_{j k} \cdot \prod_{i=1}^{N}\left[\min \left(p_{i}, q_{i}\right)-a_{i}\right]+\int_{A(p)} \alpha_{j} d \mu_{N} \cdot \int_{A(q)} \alpha_{k} d \mu_{N}\right. \\
\left.\quad+\int_{A(p)} \alpha_{k} d \mu_{N} \cdot \int_{A(q)} \alpha_{j} d \mu_{N}\right\} .
\end{aligned}
$$

$\mathrm{Use}_{d}^{\pi}$ this result in (5.2) and then rearrange terms to get

$$
\begin{aligned}
4 I_{m, n}= & \sum_{k=m}^{n} \int_{Y_{N}^{2}} h(p) \alpha_{k}(p) h(q) \alpha_{k}(q) \prod_{i=1}^{N}\left[\min \left(p_{i}, q_{i}\right)-a_{\boldsymbol{i}}\right] d \mu_{\mathbf{2 N}} \\
& +\int_{Y_{N}} h(p)\left[\sum_{j=m}^{n} \alpha_{j}(p) \int_{A(p)} \alpha_{j}(s) d \mu_{N}(s)\right] d \mu_{N}(p) \\
& \cdot \int_{Y_{N}} h(q)\left[\sum_{k=m}^{n} \alpha_{k}(q) \int_{A(q)} \alpha_{k}(s) d \mu_{N}(s)\right] d \mu_{N}(q) \\
& +\sum_{j, k=m}^{n} \int_{Y_{N}^{2}} h(p) \alpha_{j}(p) h(q) \alpha_{k}(q)\left(\int_{A(p)} \alpha_{k} d \mu_{N}\right) \\
& \cdot\left(\int_{A(q)} \alpha_{j} d \mu_{N}\right) d \mu_{2 N} .
\end{aligned}
$$

If we use "integration by parts" repeatedly to eliminate each factor $\left[\min \left(p_{i}, q_{i}\right)-a_{i}\right]$, we obtain 


$$
\begin{aligned}
\int_{Y_{N}^{2}} h(p) \alpha_{k}(p) h(q) \alpha_{k}(q) & \prod_{i=1}^{N}\left[\min \left(p_{i}, q_{i}\right)-a_{i}\right] d \mu_{2 N} \\
& =\int_{Y_{N}}\left[\int_{B(q)} h(p) \alpha_{k}(p) d \mu_{N}(p)\right]^{2} d \mu_{N}(q) \\
& =\int_{Y_{N}}\left[\int_{Y_{N}} h(p) \alpha_{k}(p) \chi_{B(q)}(p) d \mu_{N}(p)\right]^{2} d \mu_{N}(q),
\end{aligned}
$$

where $B(q)=\prod_{i=1}^{N}\left[q_{i}, b_{i}\right]$ and $\chi_{B(q)}(p)$ the characteristic function of $B(q)$. Hence on applying monotone convergence theorem and Parseval's equation, we get

$$
\begin{aligned}
\sum_{k=1}^{\infty} \int_{Y_{N}^{2}} h(p) \alpha_{k}(p) h(q) \alpha_{k}(q) & \prod_{i=1}^{N}\left[\min \left(p_{i}, q_{i}\right)-a_{i}\right] d \mu_{2 N} \\
& =\int_{Y_{N}}\left\{\int_{Y_{N}}\left[h(p) \chi_{B(q)}(p)\right]^{2} d \mu_{N}(p)\right\} d \mu_{N}(q) \\
& =\int_{Y_{N}}\left\{\int_{B(q)} h^{2}(p) d \mu_{N}(p)\right\} d \mu_{N}(q) \\
& =\int_{Y_{N}} h^{2}(p) \prod_{i=1}^{N}\left(p_{i}-a_{i}\right) d \mu_{N}(p) .
\end{aligned}
$$

Using the function $\xi$ defined by (5.3), we may write

$$
\begin{aligned}
\sum_{j, k=1}^{\infty} \int_{Y_{N}^{2}} h(p) \alpha_{j}(p) h(q) \alpha_{k}(q)\left(\int_{A(p)} \alpha_{k} d \mu_{N}\right)\left(\int_{A(q)} \alpha_{j} d \mu_{N}\right) d \mu_{2 N} \\
=\sum_{j, k=1}^{\infty} \int_{Y_{N}^{2}} h(p) \xi(p, s) \alpha_{j}(p) \alpha_{k}(s) d \mu_{2 N} \\
\\
\cdot \int_{Y_{N}^{2}} h(q) \xi(q, s) \alpha_{k}(q) \alpha_{j}(s) d \mu_{2 N} \\
=\int_{Y_{N}^{2}} h(p) \xi(p, s) h(s) \xi(s, p) d \mu_{2 N}=0,
\end{aligned}
$$

where the expression, second from the last, follows by Parseval's equation, and the last follows from the fact that $\xi(p, s) \cdot \xi(s, p)=0$ a.e. on $Y_{N}^{2}$. Use $(2.1),(5.6)$ and (5.7) in (5.5) to conclude that $\lim _{m, n} I_{m, n}=0$, thus proving the theorem. Furthermore, if we take $m=0$ in $I_{m, n}$, 


$$
\begin{aligned}
& \lim _{n} E_{N}^{f}\left[\int_{Y_{N}}(h f)_{n} d f\right]^{2} \\
(5.8) \quad & =\lim _{n} I_{0, n} \\
& =\frac{1}{4}\left[\int_{Y_{N}} h^{2}(p) \prod_{i=1}^{N}\left(p_{i}-a_{i}\right) d \mu_{N}(p)+4^{-N}\left(\int_{Y_{N}} h d \mu_{N}\right)^{2}\right] .
\end{aligned}
$$

6. Proof of Theorem 2. Let $S$ be the set of all $(f, g) \in C_{N} \times C_{N}$ for which $\int_{Y_{N}} h g d^{*} f$ exists. Then, since $h \in L^{2}$ and $g \in C_{N}$ imply $h g \in L^{2}$, it follows that the integral $\int_{Y_{N}} h g d^{*} f$ exists for almost all $(f, g)$ $\in C_{N} \times C_{N}$, i.e., $E_{N}^{o}\left\{E_{N}^{f}\left[\chi_{S}(f, g)\right]\right\}=1$, where $\chi_{S}$ is the characteristic function of $S$. Hence by Lemma 3 , it follows that

$$
E_{N}^{g}\left\{E_{N}^{f}\left[\chi_{S}(f \cos \theta-g \sin \theta, f \sin \theta+g \cos \theta)\right]\right\}=1 .
$$

By taking $\theta=\pi / 4$ in (6.1), we obtain

$$
E_{N}^{\circ}\left\{E_{N}^{f}\left[\chi_{S}\left(\frac{f-g}{\sqrt{ } 2}, \frac{f+g}{\sqrt{ } 2}\right)\right]=1,\right.
$$

from which it follows that

$$
\int_{Y_{N}} h \cdot \frac{f-g}{\sqrt{ } 2} d^{*}\left(\frac{f+g}{\sqrt{ } 2}\right)
$$

exists for a.a. $(f, g) \in C_{N} \times C_{N}$. Using the definition of the integral we may rewrite this in the form

$$
\lim _{n} \frac{1}{2} \int_{Y_{N}}[h \cdot(f-g)]_{n} d(f+g) \text { exists for a.a. }(f, g) \in C_{N} \times C_{N},
$$

where $[h \cdot(f-g)]_{n}$ is as in Theorem 1 . Similarly $\theta=-\pi / 4$ yields

(6.3) $\lim _{n} \frac{1}{2} \int_{Y_{N}}[h \cdot(f+g)]_{n} d(-f+g)$ exists for a.a. $(f, g) \in C_{N} \times C_{N}$.

Subtracting (6.3) from (6.2), we find that

(6.4) $\lim _{n}\left[\int_{Y_{N}}(h f)_{n} d f-\int_{Y_{N}}(h g)_{n} d g\right]$ exists for a.a. $(f, g) \in C_{N} \times C_{N}$.

Hence we can choose a fixed $g_{0} \in C_{N}$ such that 


$$
\lim _{n}\left[\int_{Y_{N}}(h f)_{n} d f-\int_{Y_{N}}\left(h g_{0}\right)_{n} d g_{0}\right] \text { exists for a.a. } f \in C_{N} .
$$

Thus, if we show that $\lim _{n} \int_{Y_{N}}\left(h g_{0}\right)_{n} d g_{0}$ exists, then $\lim _{n} \int_{Y_{N}}(h f)_{n} d f$ will exist for a.a. $f \in C_{N}$ and hence the proof will complete.

To show the existence of $\lim _{n} \int_{Y_{N}}\left(h g_{0}\right)_{n} d g_{0}$, we assume the opposite. Then there exist a positive $\epsilon$ and $\left\{\left(m_{i}, n_{i}\right)\right\}$ of positive integers, with $m_{i}, n_{i} \rightarrow \infty$ as $i \rightarrow \infty$, such that

$$
\int_{Y_{N}}\left(h g_{0}\right)_{n_{i}} d g_{0}-\int_{Y_{N}}\left(h g_{0}\right)_{m_{i}} d g_{0} \geqq \epsilon, \quad i=1,2, \ldots .
$$

Now,

$$
\begin{aligned}
\underset{i}{\liminf }\left[\int_{Y_{N}}(h f)_{n_{i}} d f-\int_{Y_{N}}(h f)_{m_{i}} d f\right] \\
\underset{i}{\geqq \liminf }\left\{\left[\int_{Y_{N}}(h f)_{n_{i}} d f-\int_{Y_{N}}\left(h g_{0}\right)_{n_{i}} d g_{0}\right]\right. \\
\left.\quad-\left[\int_{Y_{N}}(h f)_{m_{i}} d f-\int_{Y_{N}}\left(h g_{0}\right)_{m_{i}} d g_{0}\right]\right\} \\
+\underset{i}{\liminf }\left[\int_{Y_{N}}\left(h g_{0}\right)_{n_{i}} d g_{0}-\int_{Y_{N}}\left(h g_{0}\right)_{m_{i}} d g_{0}\right] .
\end{aligned}
$$

Use (6.5) and (6.6) in (6.7) to obtain

$$
\underset{i}{\liminf }\left[\int_{Y_{N}}(h f)_{n_{i}} d f-\int_{Y_{N}}(h f)_{m_{i}} d f\right] \geqq \epsilon \quad \text { for a.a. } f \in C_{N} .
$$

On the other hand by Theorem $1, \lim _{n} \int_{Y_{N}}(h f)_{n} d f$ exists in $L^{2}$-sense on $C_{N}$, thus giving

$$
\lim _{i} E_{N}^{f}\left[\int_{Y_{N}}(h f)_{n_{i}} d f-\int_{Y_{N}}(h f)_{m_{i}} d f\right]^{2}=0 .
$$

On using Fatou's lemma in (6.9), we see that

$$
\liminf _{i}\left[\int_{Y_{N}}(h f)_{n_{i}} d f-\int_{Y_{N}}(h f)_{m_{i}} d f\right]^{2}=0 \quad \text { for a.a. } f \in C_{N} .
$$

This is a contradiction to (6.8), thus completing the proof. 
The corollary is a simple result of Theorems 1 and 2 together with (5.8). The proof of Theorem 3 is rather lengthy and can not be included in this paper. However, the proof for the case $N=2$ is available in [2], and general cases follow in similar manner.

7. Proof of Theorem 4. Let $\left\{\alpha_{k}\right\}$ be a C.O.N. set for the definition of $\int_{Y_{N}} h d^{*} f$. Let $e_{k}=\int_{Y_{N}} h \alpha_{k} d \mu_{N}, k=1,2, \cdots$. Then by the definition of P.W.Z. integral we have

$$
\begin{aligned}
\int_{Y_{N}} h d^{*} f & =\lim _{n} \int_{Y_{N}}\left(\sum_{k=1}^{n} e_{k} \alpha_{k}\right) d f \\
& =\sum_{k=1}^{\infty}\left(\int_{Y_{N}} h \alpha_{k} d \mu_{N}\right)\left(\int_{Y_{N}} \alpha_{k} d f\right) .
\end{aligned}
$$

But $\int_{Y_{N}} \alpha_{k} d f$ is an ordinary Riemann-Stieltjes integral, and by the assumption on $f$ we have $\int_{Y_{N}} \alpha_{k} d f=\int_{Y_{N}} \alpha_{k} \cdot\left(\partial^{N} f / \partial p_{1} \cdots \partial p_{N}\right) d \mu_{N}$. (This identity may be found on p. 668 in [8].) Upon using Parseval's equation, we obtain

$$
\begin{aligned}
\int_{Y_{N}} h d^{*} f & =\sum_{k=1}^{\infty}\left(\int_{Y_{N}} h \alpha_{k} d \mu_{N}\right)\left[\int_{Y_{N}} \alpha_{k} \cdot\left(\partial^{N} f / \partial p_{1} \cdots \partial p_{N}\right) d \mu_{N}\right] \\
& =\int_{Y_{N}} h \cdot\left(\partial^{N} f / \partial p_{1} \cdots \partial p_{N}\right) d \mu_{N}
\end{aligned}
$$

which was to be shown.

The proof of Theorem 5 is similar to that of Theorem 1.

8. Proof of Theorem 6. (i) Choose $h=1$ in Theorem 5 to get

$$
\int_{0}^{1} f d^{*} f=\frac{1}{2} \int_{0}^{1} d\left[f^{2}(t)\right]=\frac{1}{2} f^{2}(1) \quad \text { for a.a. } f \in C_{W} .
$$

On the other hand, if we use the definition directly with $\left\{\beta_{k}\right\}$ $=\left\{2^{1 / 2} \sin k \pi t\right\}$, it follows that

$$
\begin{aligned}
\int_{0}^{1} f d^{*} f & =\sum_{k=1}^{\infty} b_{k} \int_{0}^{1} 2^{1 / 2} \sin k \pi t d f(t) \\
& =\sum_{k=1}^{\infty} b_{k}\left[-\int_{0}^{1} f(t) k \pi 2^{1 / 2} \cos k \pi t d t\right] \\
& =-\pi \sum_{k=1}^{\infty} k a_{k} b_{k} .
\end{aligned}
$$


Hence (i) follows from (8.1) and (8.2).

(ii) By the use of C.O.N. cosine functions $\left\{1,2^{1 / 2} \cos k \pi t\right\}$ for the definition of P.W.Z. integral, we find that

$$
\begin{aligned}
\int_{0}^{1} f d^{*} f= & \int_{0}^{1} f(t) d t \cdot \int_{0}^{1} d f(t)+\sum_{k=1}^{\infty} a_{k} \int_{0}^{1} 2^{1 / 2} \cos k \pi t d f(t) \\
= & f(1) \int_{0}^{1} f(t) d t \\
& +\sum_{k=1}^{\infty} a_{k}\left[f(1) 2^{1 / 2} \cos k \pi+k \pi \int_{0}^{1} f(t) 2^{1 / 2} \sin k \pi t d t\right] \\
= & f(1) \int_{0}^{1} f(t) d t+2^{1 / 2} f(1) \sum_{k=1}^{\infty}(-1)^{k} a_{k}+\pi \sum_{k=1}^{\infty} k a_{k} b_{k} .
\end{aligned}
$$

Substituting (i) in (8.3) and then using the fact that $f(1)=0$ for only a set of functions of Wiener measure zero in $C_{W}$, we finally obtain the result (ii).

(iii) We now use the C.O.N. trigonometric functions $\left\{1,2^{1 / 2} \sin 2 k \pi t, 2^{1 / 2} \cos 2 k \pi t\right\}$ for $\int_{0}^{1} f d^{*} f$ to get

$$
\begin{aligned}
\int_{0}^{1} f d^{*} f= & \int_{0}^{1} f(t) d t \cdot \int_{0}^{1} d f(t) \\
& +\sum_{k=1}^{\infty}\left[b_{2 k} \int_{0}^{1} 2^{1 / 2} \sin 2 k \pi t d f(t)\right. \\
& \left.+a_{2 k} \int_{0}^{1} 2^{1 / 2} \cos 2 k \pi t d f(t)\right] .
\end{aligned}
$$

After using "integration by parts" for the R-S integral on the right member of (8.4), we find that

$$
\int_{0}^{1} f d^{*} f=f(1) \int_{0}^{1} f(t) d t+2^{1 / 2} f(1) \sum_{k=1}^{\infty} a_{2 k}
$$

Substitution of (8.1) in (8.5) yields (iii).

(iv) Now, from (ii) and (iii)

$\sum_{k=1}^{\infty} a_{k}=2 \sum_{k=1}^{\infty} a_{2 k}-\sum_{k=1}^{\infty}(-1)^{k} a_{k}=-2^{-1 / 2} \int_{0}^{1} f(t) d t \quad$ for a.a. $f \in C_{W}$.

The corollary is a simple result of (iv). 


\section{BIBLIOGRAPHY}

1. R. E. A. C. Paley, N. Wiener and A. Zygmund, Noles on ranảom functions, Math. Z. 37 (1933), 647-668.

2. C. Park, Generalized Riemann-Stieltjes integral over the K. Y. W. space of functions of two variables, Thesis, Univ. of Minnesota, 1968.

3. J. Kuelbs, A Cameron-Martin translation theorem for a Gaussian measure on $C(Y)$, Proc. Amer. Math. Soc. 19 (1968), 109-114.

4. H. D. Colson, An existence theorem for a generalized Riemann-Stieltjes integral, Thesis, Univ. of Minnesota, (1949).

5. J. Yeh, Cameron-Martin translation theorems in the Wiener space of functions of two variables, Trans. Amer. Math. Soc. 107 (1963), 409-420.

6. - Orthogonal development of functionals and related theorems in the IViener sbace of functions of two variables, Pacific J. Math. 13 (1963), 1427-1436.

7. J. E. Bearman, Rotations in the product of two Wiener spaces, Proc. Amer. Math Soc. 3 (1952), 129-137.

8. E. W. Hobson, The theory of functions of a real variable and the theory of Fonirier's series. Vol. I, Dover, New York (1957).

9. A. Wilansky, Functional analysis, Blaisdell, Waltham, Mass., (1964).

\section{Miami University}

